\title{
LEONES, CAZADORES E HISTORIADORES, A PROPÓSITO DE LAS POLÍTICAS DE LA MEMORIA Y DEL CONOCIMIENTO
}

\author{
POR \\ Hugo Achugar \\ Universidad de la República, Montevideo
}

Hay un proverbio africano que dice: "Hasta que los leones tengan sus propios historiadores, las historias de cacería seguirán glorificando al cazador" (Eduardo Galeano). El proverbio escenifica un conflicto permanente mediante tres personajes: leones, cazadores e historiadores, o dicho de otra manera, los oprimidos, los opresores y los intelectuales. $\mathrm{Al}$ mismo tiempo que alude a una historia, diseña dos lugares y dos prácticas intelectuales: el lugar y la acción de los leones y el lugar y la acción de los cazadores.

Hay otra historia, de origen brasileño, que ofrece una variante de interés: un hombre narra a un amigo su aventura con una onza. A medida que avanza el relato, el oyente interfiere reiteradamente en el relato lo que obliga al fastidiado narrador a preguntar: "¿Vocé é amigo mio u da onza?"

La historia de la onza agrega un personaje o una situación al escenario del proverbio africano: se trata del intelectual que sin ser onza o león es, sin embargo, amigo de la onza. $\mathrm{O}$ formulado de otro modo, lo que se agrega es la posicionalidad del intelectual que sin pertenecer al ámbito de los oprimidos leones se ubica a su lado y toma, sino una "identidad prestada", una "conciencia de onza prestada".

El relato construido por el proverbio africano y la anécdota brasileña bien podría ser considerado como una descripción del escenario contemporáneo en relación con varios de los debates del presente fin de siglo. Así, la discusión en torno a las identidades en relación con el intelectual, la nación, la región y el proceso de globalización parece centrarse en el tema de la posicionalidad. Pero también, como el mismo relato lo indica, supone el debate sobre el propio relato historiográfico y sobre las localizaciones de la memoria. Incluso, supone el debate en torno al estatuto tanto de la memoria oficial como de la memoria colectiva, de la memoria desde el poder como de la memoria desde los oprimidos; dicho sea de paso, es en la formulación uruguaya de ese debate y su relación con la tortura y los desaparecidos que Eduardo Galeano recupera el proverbio africano. Posicionalidad, locación y memoria, entonces, son los centros del debate político e intelectual de este fin de siglo.

Ahora bien, ¿qué se está discutiendo en este fin de siglo? Al parecer, una vez más, los intelectuales están debatiendo en torno al poder y en torno al poder de la representación. Pero ¿qué es lo que realmente se disputa cuando se debate en torno al poder y a la representación? La respuesta varía según el lugar y el idioma desde donde se la formule o según el lugar y el idioma desde donde se la escuche. 
Pero ¿cuál es la relación entre el debate de la academia y el debate de la sociedad civil? y ¿cómo es esa relación en los Estados Unidos y en América Latina? ¿Es el mismo debate el que se desarrolla en América Latina y el que se desarrolla en los Estados Unidos y en gran parte de la academia del primer mundo? O incluso, ¿cómo se relaciona el debate sobre la globalización, el estado-nación, la integración regional, con el debate sobre los llamados estudios postcoloniales y los subalternos de la academia del primer mundo? Y también ¿cuáles son los personajes que entran en estos debates? Parece claro que entre los sujetos implicados se encuentran los intelectuales, además del Estado, los migrantes, las distintas minorías, a saber: las determinadas por género, etnia, preferencia sexual, así como los tradicionalmente determinados por la clase socio-económica. De igual manera, las instituciones generadoras de opinión y conocimiento como los medios de comunicación y las universidades tienen un presencia en el debate. En resumen, ¿en qué medida y cómo se relacionan los múltiples debates en el escenario del presente fin de siglo? Y ¿cómo se posicionan frente a esos debates los intelectuales o los académicos en América Latina y en los EE.UU.? En esta oportunidad no voy a discutir todos los temas aludidos y me voy a limitar solamente a algunos aspectos de la función de la nación, la migración y los intelectuales en el actual proceso de integración de globalización.

\section{El lugar desde donde se lee. A propósito de la nueva construcción teórica de AMÉRICA LATINA}

La discusión sobre la posicionalidad no es nueva aunque presente algunas diferencias con respecto al debate de hace unas décadas. La localización y el posicionamiento de la enunciación y del conocimiento es radicada - al menos por una parte de la academia del primer mundo y del Commonwealth - en la discusión del binarismo colonizado/colonizador o hegemónico/subalterno aun cuando se sostenga que las fronteras de dichos binarismos fueron redibujadas y la división colonizador/colonizado fue reordenada (Prakash 3). ${ }^{1}$ El intento de liberar la historiografía de la dominación de categorías e ideas producidas por el colonialismo, a saber colonizador/colonizado, blancos, negros y café con leche, civilizados y bárbaros, modernos y arcaicos, identidad cultural, tribu y nación (Prakash 5) guía parte de esta discusión. Esto que se presenta como una novedad, sin embargo, existe en el pensamiento latinoamericano desde hace mucho tiempo; en realidad, precede la actual discusión — según el relato que se prefiera - desde hace varios siglos o desde hace más de un siglo. Y cuando se argumenta que: "it should be recognized that while there are many examples of critiques of liberal historiography and of its complicity with imperialism, the revision of the discipline from the place of otherness is yet to occur" (Prakash 5) me pregunto ¿dónde es que está por ocurrir? De ser así, ¿cómo leer el revisionismo histórico

\footnotetext{
' Como señala Gyan Prakash: "Modern colonialism, it is now widely recognized, instituted enduring hierarchies of subjects and knowledges - the colonizer and the colonized, the Occidental and the Oriental, the civilized and the primitive, the scientific and the superstitious, the developed and the underdeveloped. The scholarship in different disciplines has made us all too aware that such dicotomies reduced complex differences and interactions to the binary (self/other) logic of colonial power" (3).
} 
latinoamericano durante el siglo XX? O ¿cómo leer la labor de José Martí sino como la revisión histórico-cultural desde la perspectiva del otro que es el latinoamericano? ${ }^{2}$

En gran parte del pensamiento originado en el marco del Commonwealth teórico poscolonialista se ignora la producción latinoamericana o, en el mejor de los casos, se procede a analizar América Latina como un conjunto homogéneo derivado de un pasado histórico colonial supuestamente común en lo esencial con India, Africa y otras regiones del planeta. No voy a reiterar aquí mi cuestionamiento de la construcción que se ha estado haciendo de América Latina y del nosotros latinoamericano (Achugar, en prensa) pero quisiera insistir en señalar uno de los mayores equívocos en el tratamiento de América Latina que es el de su homogeneización o el de su reducción como epítome de lo poscolonial o de lo subalterno. América Latina es uno de los campos de batalla en donde los distintos sujetos combaten por la construcción de su proyecto en función de sus particulares memorias. En ese sentido, América Latina operaría del mismo modo que, según Prasentij Duara, funciona la nación, es decir como el espacio donde combaten por el poder distintos proyectos nacionales y del mismo modo que, según Claudia Koonz, opera la memoria pública; es decir, como el lugar donde distintas memorias compiten por el poder.

Por lo mismo, tanto la evaluación de los distintos pasados como la propuesta de los diversos futuros y el posicionamiento en relación con el poder determinan el tipo o los tipos de América Latina que permiten construir los respectivos nosotros inclusivos y excluyentes desde los que se habla (Ticio Escobar en Gerardo Caetano). ${ }^{3}$ Nada de esto es novedad pero se vuelve particularmente relevante cuando se trata de determinar o de proponer políticas del conocimiento o agendas teóricas que postulan la construcción de América Latina sin atender a sus respectivas especificidades históricas y culturales asimilándola a la experiencia histórica de lo acontecido en los países que integraron el Imperio británico y formaron parte del Commonwealth.

En ese sentido, la construcción que se propone de América Latina, dentro del marco teórico de los llamados estudios postcoloniales, parecería apuntar a que el lugar desde donde se habla no es o no debería ser el de la nación sino el del pasado colonial. Lo que no parece tenerse en cuenta es que esa construcción de América Latina, como toda construcción, supone además del lugar desde donde se habla, el lugar desde donde se lee. Y, precisamente, el lugar desde donde se lee América Latina parece ser, por un lado, el de la experiencia histórica del Commonwealth y por otro, como veremos más adelante, el de la agenda de la academia norteamericana que está localizada en la historia de su sociedad civil.

Tal parece que los académicos del movimiento poscolonial, anclados en una memoria escrita o dicha en inglés -en particular en relación con los estudios culturales o literariosdescubrieron que la fragmentación que ellos realizaban en British, American, Austrialian, Indian, Asian, Caribbean y African English estaba basada en una estructura colonial, más aún descubrieron - por la migración de intelectuales English speaking a los centros académicos del primer mundo- que había perdido sentido el estudio de las culturas

\footnotetext{
${ }^{2}$ Soy consciente que el uso tanto de la noción del "otro" como la de "latinoamericano" plantean muchos problemas. Sin embargo, su presencia en este texto no surge de una posición "neo-criollista" o "fundamentalista", posición que no comparto ni de la del "subalternismo" con la que tengo particulares diferencias. En un próximo ensayo daré cuenta de los problemas aquí apenas aludidos. ${ }^{3}$ La referencia a Ticio Escobar está en Hugo Achugar y Gerardo Caetano.
} 
nacionales y que debían ser sustituidas por categorías como Global English (Rosemary George); dicho sea de paso, ese tipo de categorías resuenan en oídos hispanoamericanos de un modo inquietantemente similar al de los proyectos "hispanófilos" propios del intento nostálgico-restaurador del franquismo. Por otra parte, como el modelo se ajustaba al presente período de globalización económico-financiera entendieron que se podía extender sin más al conjunto del planeta. No tuvieron en cuenta que América Latina -0 , a los efectos, Iberoamérica - funciona como categoría del conocimiento, por lo menos, desde hace más de un siglo y que tanto la revisión como la crítica de dicha noción ha sido y es constante. No tuvieron en cuenta, además, que la conciencia latinoamericana articula varias inscripciones nacionales y que la "patria grande" podía albergar múltiples "patrias chicas". No tuvieron en cuenta, por último, que la conciencia latinoamericana ha sido desde hace siglos un espacio heterogéneo donde los distintos sujetos sociales, étnicos y culturales han venido batallando por construir sus respectivos proyectos sociales y culturales. En ese sentido, lo que el pensamiento poscolonial generado en los ámbitos académicos pertenecientes o actuantes en el Commonwealth no parece haber considerado es que el permanente cuestionamiento de los latinoamericanos en relación con su identidad tiene que ver, precisamente, con el hecho de haber sido y de seguir siendo exactamente eso; es decir, un espacio heterogéneo en constante transformación donde ninguna identidad global es permanente o aceptada de modo general. Olvidaron o no tuvieron en cuenta que la resistencia a las distintas construcciones que sobre ella se realizaban tenía que ver con porcesos históricos específicos y que el proyecto de configuración o construcción de una América Latina unida tiene una larga, polémica y conflictiva historia.

La globalización leída desde el Commonwealth parece no haber abandonado ciertos hábitos y ciertas memorias de la historia imperial en lengua inglesa. La lectura desde el Commonwealth, como toda mirada - voluntaria o involuntariamente autocentrada, puede ignorar y construir su interpretación mediante el recorte y la mezcla sin preocuparse de la operación ideológica que realiza. ${ }^{4}$ Pero claro, para ciertas miradas aun los textos imperiales o canónicos escritos en otras lenguas son pasibles de ser leídos como ejemplos de resistencia a la colonización sin considerar la situación histórica en que dichos textos fueron producidos. Y además, una cosa es ser poscolonial en inglés y otra en español, portugués, bayano, quechua, aymara, guaraní, papiamento y equivalentes.

Lo preocupante, sin embargo, no es la manipulación y la tergiversación. Después de todo en la periferia estamos acostumbrados a este tipo de relatos; más aún, desde Guaman Poma tenemos ideas de lo que es memoria oficial y contramemoria. El problema es que en la fundamentación de esta historiografía el lugar no parece importar. Eso al menos es lo que propone una amplia teorización acerca de la desterritorialización económica que se ha extendido a lo cultural. ¿No importa o lo que ocurre es que se está procediendo a una reubicación de la enunciación y de la posición o del lugar de la memoria? Lo que parece haber ocurrido es, como propone Habermas, una "reubicación de la autoridad". Esa "reubicación de la autoridad" y el modo en que esa reubicación se está procesando es

\footnotetext{
${ }^{4} \mathrm{Al}$ respecto es interesante tanto la "Introduction: The Essential Heterogeneity of Being" que realiza Anna Rutherford en From Commonwealth to Post-colonial como el ensayo de Wilson Harris "The Fabric of Imagination" donde se parafrasea Antonio Machado a través de una cita de El laberinto de la soledad de Octavio Paz.
} 
precisamente lo que ocupa el debate sobre el pasado y sobre la memoria. O dicho de otro modo, la reubicación de la autoridad es o implica la reubicación del pasado y, en consecuencia, la reubicación de la memoria colectiva. Reubicación del pasado que es también una redefinición de lo memorable y de lo olvidable.

Toda memoria, toda recuperación y representación de la memoria implica una evaluación del pasado. El tiempo de la evaluación de este fin de siglo es para unos posnacional y para otros poscolonial. Lo que no se toma en cuenta es que, si bien la globalización de la economía ha podido volver obsoleta la categoría y el ámbito del estadonación, las formaciones nacionales no se agotan en lo económico y que las múltiples historias -dominantes o silenciadas, hegemónicas o subalternas- al igual que las múltiples memorias son un elemento central de la categoría "nación", incluso en estos tiempos globalizados; lo que no implica suscribir el proyecto decimonónico del estadonación. El lugar desde donde se lee en América Latina está nutrido por múltiples memorias que se llaman Guamán Poma, Atahualpa, el Inca Garcilaso, Simón Bolívar, José Gervasio Artigas, Martí, Hostos, Mariátegui, Torres García, y otro muchos más que no se agotan en dos o tres hot commodities puestas a circular en el mercado globalizado como parece haber ocurrido con Rigoberta Menchú. ${ }^{5}$ Incluso, cuando las memorias particulares o subregionales puedan sustituir Guaman Poma por Gardel, Hostos por Daniel Santos o Mariátegui por Tiradentes o todavía más, aun cuando la memoria esté signada por Perón, Rosas o Getulio Vargas. Lo que estoy argumentando es que el paisaje que diseñan esas múltiples memorias suponen un posicionamiento y un lugar desde donde se habla y desde donde se lee lo que es específico y que ese lugar está marcado por procesos históricos que no son borrados inexorablemente por la globalización.

Por otra parte, el marco teórico de los estudios poscoloniales que intenta construir un supuesto nuevo lugar desde donde leer y dar cuenta de América Latina no sólo no toma en consideración toda una memoria y una tradición de lectura sino que además aspira a presentarse como algo distinto de lo realizado en nuestra América. Ese nuevo marco teórico se ha llamado de múltiples modos en América Latina. Se ha llamado "pensamiento latinoamericano" y, bien o mal, ha estado detrás de la discusión que, por lo menos, desde Andrés Bello hasta nuestros días recorre el debate latinoamericano. Más aún, ha tenido distintas versiones y ha podido, como decía Martí, albergar tanto aquéllos que intentaban seguir viviendo la Colonia como aquéllos que, como el propio Martí, construyeron "nuestra" visión. Una visión que ha permitido dar cuenta de la historia sin necesidad de un supuesto nuevo marco teórico que, insisto, supuestamente podría dar cuenta de fenómenos que eran invisibles para los propios latinoamericanos cosa que la historia del pensamiento latinoamericano muestra no ser así. Una visión, a la vez, moderna y posmoderna y que surge de la historia de las agendas de la sociedad latinoamericana.

\footnotetext{
${ }^{5}$ La referencia a Rigoberta Menchú no supone un juicio sobre ella ni sobre su testimonio Me llamo Rigoberta sino sobre la utilización reductora que de ambos se ha hecho en el "mercado" internacional; especialmente en el académico.
} 


\section{A PROPÓSITO DE MIGRACIÓN Y MELTING POTS}

Uno de los lugares comunes de la actual discusión se refiere a las transformaciones producidas por las migraciones - especialmente el fenómeno de la migración sur/norteen las sociedades contemporálneas. En ese sentido, gran parte de la reflexión refiere al impacto que la diversidad étnico, religiosa y cultural ha producido al interior de países hasta no hace mucho mono-culturales. Esto - junto con el flujo económico-financiero de capitales - habría producido sociedades multiculturales y habría terminado por liquidar la vigencia del estado-nación decimonónico. La caracterización parece no ofrecer mayores problemas en relación con muchos países del llamado primer mundo y en especial con los EE.UU. Pero, ¿sucede lo mismo en todas partes?; y lo que es más significativo: ¿el proceso de discusión o de desconstrucción del llamado melting-pot o "crisol de razas" en países como los EE.UU. es similar al de la totalidad de América Latina?

¿Cúal es la agenda que determina la pertinencia o la legitimidad de los conocimientos? ¿Las agendas fundadas en las batallas que las minorías llevan adelante en la sociedad norteamericana o las agendas diseñadas en el seno de la sociedad latinoamericana? La agenda de las sociedades del primer mundo regula el pasado de acuerdo con la lucha que los distintos sujetos sociales - nuevos y tradicionales - llevan adelante en la conformación de sus respectivas sociedades civiles. Esa agenda es legítima pero no es universal a no ser que, en virtud de la globalización, se la quiera globalizar y exportar. En ese sentido lo planteado por George Yúdice en relación con el arte y la producción latinoamericanos acerca de la importancia de la intermediación o del brokering ejercida en la sociedad norteamericana respecto de lo latinoamericano adquiere un particular interés. Es, precisamente, esa intermediación, ese brokering pero referido a la producción de conocimientos lo que estoy cuestionando. Pues, como dice Yúdice, lo latinoamericano se confunde a menudo con lo "latino-estadounidense" en el proyecto multicultural de transformar la sociedad civil estadounidense. Este proyecto está:

sobredeterminado tanto por una nueva forma de democratización cultural, basada en las demandas de "grupos de identidad", como por la necesidad de la cultura estadounidense de proyectarse como si fuese "isomorfa" con el mundo, es decir, como microcosmo del mundo, característica que legitima su estatus como única superpotencia ... (174).

O como dice en otro pasaje:

Si ante se le pedía al latinoamericano que escenificara su surrealismo nato, a lo Carpentier, o su realismo maravilloso, o su macondismo, hoy día se le pide que se convierta en un casi chicano o latino (150).

Intermediación o brokering del conocimiento basado en la agenda de la sociedad civil estadounidense y que incluye una reformulación del espacio. Así, la frontera puede ser un espacio legítimo y de particular relevancia para sujetos sociales o para individuos como los teorizados por Gloria Anzaldúa, pero eso y el hecho de que Anzaldúa tenga cierta relación con la herencia latina no habilita a proponer las memorias y la situación de los latino- 
estadounidenses como válida para el conjunto de los latinoamericanos, ni tampoco a que latinoamericanos y latino-estadounidenses sean identificados sin más entre sí.

El reconocimiento de la heterogeneidad de América Latina así como la del resto del planeta no parece ser mantenido cuando se propone el paradigma de lo poscolonial para la historia socio-cultural latinoamericana. ¿Por qué no reconocer que las políticas de la memoria y del conocimiento están ligadas al lugar desde donde se habla y desde donde se lee y que los problemas de ese conjunto — para nada homogéneo- que son los llamados "latinos" en los EE.UU. no son necesariamente los del heterogéneo conjunto llamado "latinoamericanos" viviendo en sus respectivos países o incluso migrados a otros países de América Latina? ¿Porquéno pensar que con similares herencias - aunque no necesariamente con iguales memorias o con iguales historias - pueden haber diversos sujetos de conocimiento? Más aún, ¿por qué no pensar que la migración, el trasterramiento y la inserción en un nuevo lugar desde donde se habla, produce conocimientos diferentes? Y por último, ¿por qué no pensar que la historia de los intentos de melting pot o "crisol de razas" no es la misma en los EE.UU. y en distintas partes de América Latina? ¿Por qué no pensar que la lucha por los derechos civiles alteró el proyecto del melting pot en los EE.UU. y que en América Latina el proyecto del "crisol de razas" ha tenido una historia distinta y que además varía según las regiones? Y para terminar, ¿me pregunto si el fracaso o la erosión del proyecto del melting pot en los EE.UU. obliga a una idéntica lectura de la historia del proyecto del "crisol de razas" en América Latina?

Aunque más prudente sería señalar que al parecer desde o en Uruguay —o desde el Río de la Plata y hasta desde el sur de Brasil- los términos de la discusión acerca del poscolonialismo - y también de la subalternidad - no tienen el sentido que la academia norteamericana o el nuevo Commonwealth teórico piensa que tiene o que debería tener para el conjunto de América Latina. Entre otras razones por las que ofrece Jorge Klor de Alva cuando dice:

It is misleading to characterize the Americas following the civil wars of separation as postcolonial. In short, the Americas were neither Asia nor Africa; Mexico is not India, Peru is not Indonesia, and Latinos in the US - although tragically opposed by an exclusionary will- are not Algerians (3). ${ }^{\text {? }}$

A lo que quizás cabría agregar que si bien los Latinos in the US no son argelinos, tampoco son latinoamericanos y por último que, a diferencia de la India, las culturas de las varias América Latina no forman parte del Commonwealth a pesar de la globalización; es decir, no forman parte de una memoria histórica signada por la cultura en inglés del

\footnotetext{
${ }^{7}$ Lo planteado por Jorge Klor de Alva en este artículo, "Colonialism and Postcolonialism as (Latin) American Mirages" se enriquece con lo que plantea en "The Postcolonization of the (Latin) American Experience: A Reconsideration of 'Colonialism', 'Postcolonialism', and 'Mestizaje'”. Del mismo modo, lo planteado en el presente ensayo es una versión no sólo preliminar sino de "barricada" que en la versión final ofrece una consideración más detenida. En particular en relación con la noción de Klor de Alva (1995) que sostiene "... postcoloniality can best be thought of as a form of constetatory/ oppositional conciousness, emerging from either preexisting imperial, colonial or ongoing subaltern conditions, which fosters processes aimed at revising the norms and practices of antecedent or still
} 
Commonwealth. O dicho de otra manera, que podemos dar cuenta de los fenómenos histórico-culturales de América Latina - tanto de su presente como de su pasado- desde marcos teóricos como los contenidos en categorías como las de nuesıra América y otras desarrolladas desde el pensamiento latinoamericano.

Para terminar y seguramente para abrir otro aspecto del debate, ¿en qué medida las propuestas que formulan estas nuevas agendas "panamericanistas" contenidas en mucha de la reflexión surgida desde algunos sectores de la academia norteamericana o influidas por ella no están, de hecho, teorizando la constitución del bloque económico diseñado en la cumbre hemisférica de diciembre de 1994 en Miami y conocido como ALCA? Y, ¿en qué medida la situación del presente fin de siglo no vuelve a reeditar, salvando todas las diferencias y todas las distancias, las circunstancias y los debates presentes en la Conferencia convocada por Mr. Blaine en 1888 y que, entre otras razones, llevaron a Martí a formular su advertencia contenida en la expresión-consigna "nuestra América"? ¿No estaremos nuevamente, - -gracias a la reconstrucción teórica que se intenta desde el ámbito intelectual del Commonwealth y que se rearticula en los EE.UU., enfrentados a la disolución de las diferencias dentro de una globalización supuestamente más digerible pues se presenta como una versión "panamericanista" de una supuesta historia de los leones? No estaremos, sin embargo, me pregunto para concluir, simplemente en una reedición del "panamericanismo" que intenta obliterar el "nuestro americanismo" de Martí pues se trata de la propuesta de una integración regional más preocupada del fortalecimiento de bloques económicos en Europa y Asia que de los intereses latinoamericanos? El debate sigue abierto.

\section{BiBLIOGRAFÍA}

Achugar, Hugo y Gerardo Caetano (comps.). Mundo, región, aldea. Identidades políticas, culturales e integración regional. Montevideo: Ediciones Trilce, 1994.

Galeano, Eduardo. "Memorias y desmemorias". Brecha. (Montevideo, abril 4 de 1997) contratapa.

George, Rosemary Marangoly. The Politics of Home. Postcolonial relocations and Twentieth-century Fiction. Cambridge/Nueva York/Melbourne: Cambridge University Press, 1996.

Habermas, Jürgen. The Past as Future (Vergangenheit als Zakunft). Jürgen Habermas interviewed by Michael Haller, translated and edited by Max Pensky. Lincoln and London: University of Nebraska, 1994.

Klor de Alva, Jorge. "The Postcolonization of the (Latin) American Experience: A Reconsideration of 'Colonialism', 'Poscolonialism' and 'Mestizajes'”. After Colonialism, Imperial Histories and Postcolonial Displacements. Gyan Prakash, ed. Princeton: Princeton University Press, 1995. 241-275.

vital forms of domination". (245, énfasis mío). Esta perspectiva de Klor de Alva puede ser compartida y permite una comprensión de lo "poscolonial" casi similar - sino la misma - a la mantenida por toda una tradición del pensamiento latinoamericano progresista o de izquierda, lo que obliga a una discusión más detenida de la cuestión y que dadas las características del presente ensayo no son posibles en esta oportunidad. 
"Colonialism and Postcolonialism as (Latin) American Mirages". Colonial Latin American Review 1/1-2 (1992): 3-23.

Prakash, Gyan. "Introduction: After Colonialism". After Colonialism Imperial Histories and Postcolonial Displacements. Gyan Prakash (ed.). Princeton: Princeton University Press, 1995.

Rutherford, Anne (ed). From Commonwealth to Literature. Sidney: Dangaroo Press, 1992.

Yúdice, George. "Globalización y nuevas formas de intermediación cultural”. Mundo, región, aldea. Identidades politicas, culturales e integración regional. Hugo Achugar y Gerardo Caetano (comps.). Montevideo: Ediciones Trilce, 1994. 134-157. 
